\title{
AVALIAÇÃO DE TANQUES DE ESCORVA UTILIZADOS EM SUBSTITUIÇÃO À VÁLVULA-DE-PÉ EM INSTALAÇÕES DE BOMBEAMENTO ${ }^{1}$
}

\author{
MARCOS O. BETINI ${ }^{2}$, JOSÉ R. ZANINI ${ }^{3}$, LETICIA C. FORATTO ${ }^{4}$, \\ GENER T. PEREIRA ${ }^{5}$
}

\begin{abstract}
RESUMO: A maioria das instalações de abastecimento de água utiliza bombas hidráulicas do tipo turbobombas, requerendo que o interior de sua carcaça e da tubulação de sucção esteja preenchido com água (escorvados) para que a sucção da água possa ser efetivada. O escorvamento das bombas pode ser efetuado instalando-as abaixo do reservatório de captação (bomba afogada). Quando a bomba está acima do reservatório e o escorvamento é manual, é necessário usar válvulas-de-pé, que são suscetíveis ao mau funcionamento, limitando a confiabilidade do sistema, principalmente em caso de automação. Como alternativa à válvula-de-pé, neste trabalho, foram estudados dois tanques escorva, testados no Laboratório de Hidráulica e Irrigação da FCAV/UNESP - Jaboticabal, avaliando-se situações de altura manométrica de sucção de bomba centrífuga e relações entre volume útil do tanque e volume do tubo de sucção, tendo-se concluído: a) o volume do tanque de escorva é função do volume do tubo de sucção da instalação de bombeamento e da altura manométrica de sucção; b) o volume do tanque de escorva pode ser calculado seguindo-se a Lei de Boyle, com percentagem de acréscimo que, neste estudo, foi de $10 \%$ para um modelo de tanque e de $30 \%$ para o segundo modelo.
\end{abstract}

PALAVRAS-CHAVE: bombas hidráulicas, bomba não-afogada, escorvamento.

\section{EVALUATION OF PRIMING TANKS USING THEM INSTEAD OF FOOT VALVE IN PUMPING INSTALLATIONS}

\begin{abstract}
The majority of water supply installations use hydraulic pump-turbines, requiring their inner casing and suction pipe be water filled (primed), to allow water suction. Pumps priming can be carried out installing them under the suction reservoir (drowned pump). When pump is above the reservoir and priming is manual, it will be necessary using foot valves, which are subjected to bad working, with a reduction of system reliability, mainly with automation. In this work, as an alternative to foot valves, two priming tanks were inquired, tested in the FCAV/UNESP-Jaboticabal, Brazil, Laboratory of Hydraulics and Irrigation, with the evaluation of suction head situations of centrifugal pump and relations between the useful volume of tank and suction pipe volume, with the conclusion as follows: a) priming tank volume is a function of pumping installation suction pipe volume and of suction head; b) priming tank volume can be calculated according to Boyle's Law with an increase percentage, which was in this inquiry $10 \%$ for a tank model and 30\% for the second model.
\end{abstract}

KEYWORDS: hydraulic pumps, not drowned pump, priming.

\footnotetext{
${ }^{1}$ Extraído da Dissertação de Mestrado do primeiro autor.

${ }^{2}$ Engํㅡㄹ Agrônomo, mestre em Agronomia pela UNESP-Jaboticabal, bettini@com4.com.br.

${ }^{3}$ Eng ${ }^{\mathrm{o}}$ Agrônomo, Prof. Dr., Departamento de Engenharia Rural, UNESP-Jaboticabal.

${ }^{4}$ Eng ${ }^{\mathrm{a}}$ Agrônoma, graduada pela UNESP-Jaboticabal.

${ }^{5}$ Estatístico, Prof. Dr., Departamento de Ciências Exatas, UNESP-Jaboticabal.

Recebido pelo Conselho Editorial em: 4-9-2007

Aprovado pelo Conselho Editorial em 5-8-2008
} 


\section{INTRODUÇÃO}

A maioria das instalações de abastecimento de água utiliza bombas hidráulicas do tipo turbobombas, requerendo o escorvamento, que se refere ao preenchimento do interior da bomba e da tubulação de sucção com o líquido a ser bombeado para a sua partida. $\mathrm{O}$ escorvamento das bombas pode ser efetuado instalando-as abaixo do nível de água do reservatório de captação (bomba afogada); mas, para instalações com bombas não-afogadas, freqüentemente são utilizadas válvulas-de-pé (CARVALHO, 1992). Por apresentar partes móveis, a válvula-de-pé é suscetível ao mau funcionamento, limitando a confiabilidade do sistema, principalmente em caso de automação. Segundo MACINTYRE (1997), os defeitos mais comuns verificados nos bombeamentos relacionam-se com a sucção. Por isso, essa parte da instalação deve receber especial atenção.

CARVALHO (1992) comenta que, dos diversos modelos de bombas, destacam-se as hidrodinâmicas de fluxo radial, também conhecidas como bombas centrífugas. Segundo VILELA et al. (2003), quando a bomba é instalada abaixo do nível da água do reservatório, a tubulação de sucção permanece constantemente preenchida com o líquido, o que constitui uma vantagem. Porém, as dificuldades de instalação, o excesso de umidade, a maior dificuldade para a manutenção e o risco de entupimento são os maiores inconvenientes. Assim, na maioria dos sistemas de recalque, opta-se por instalar o conjunto de bombeamento acima do nível da água do reservatório de sucção.

Conforme AZEVEDO NETTO et al. (1998), antes de pôr em funcionamento as bombas centrífugas, é necessário realizar o escorvamento. Além disso, as peças dentro da bomba dependem da lubrificação que lhes é fornecida pelo líquido a deslocar. Para o escorvamento, é comum utilizar: bomba submersa, injetor, bomba de vácuo e válvula-de-pé. Segundo MACINTYRE (1997), as turbobombas não são auto-aspirantes ou auto-escorvantes, isto é, não são capazes de expulsar o ar pelo rotor, criando vácuo capaz de permitir a entrada do líquido, no início do funcionamento. Quando postas a funcionar, já devem estar cheias de líquido e, por conseguinte, também a tubulação de sucção.

Apesar de a válvula-de-pé existir em muitas instalações, ela aumenta acentuadamente a perda de carga na sucção, possui custo considerável, necessita de limpeza freqüente e representa risco em instalações com automação da partida, devido ao seu desgaste ou mau funcionamento, oriundos de partículas contidas na água.

Os tanques de escorva oferecem maior garantia de escorva do que as válvulas-de-pé, quando dimensionados e aplicados corretamente. Geralmente, são de formato cilíndrico ou ovalados, instalados vertical ou horizontalmente. O equipamento é de fácil construção e instalação, não tem partes móveis, praticamente não sofre desgastes e o custo de aquisição é semelhante ao da válvulade-pé. Dentre as limitações que oferecem, devem ser mencionados: maior peso e tamanho do que as válvulas de pé, requerendo maior área e estrutura para sua instalação, poucos fabricantes comerciais, conhecimento e informações técnicas sobre seu uso.

Segundo VILLA NOVA et al. (1997), em instalações de recalque, é freqüente o uso da tradicional válvula-de-pé, que geralmente necesssita de limpeza freqüente. Como alternativa a essa válvula, os autores propuseram a utilização de um "balão de escorva". Baseando-se na Lei de Boyle, apresentaram equação para o dimensionamento do balão, em função dos parâmetros da aspiração.

Pela lei geral dos gases perfeitos, $\left(\mathrm{P}_{1} \mathrm{~V}_{1}\right) / \mathrm{T}_{1}=\left(\mathrm{P}_{2} \mathrm{~V}_{2}\right) / \mathrm{T}_{2}$, em que: $\mathrm{P}_{1}$ - pressão do gás no estado inicial; $\mathrm{V}_{1}$ - volume do gás no estado inicial; $\mathrm{P}_{2}$ - pressão do gás no estado final; $\mathrm{V}_{2}$ - volume do gás no estado final; $\mathrm{T}_{1}$ - temperatura do gás no estado inicial, e $\mathrm{T}_{2}$ - temperatura do gás no estado final. Sendo a temperatura constante (transformacão isotérmica), verifica-se que a pressão e o volume são inversamente proporcionais, e a expressão reduz-se a: $\mathrm{P}_{1} \mathrm{~V}_{1}=\mathrm{P}_{2} \mathrm{~V}_{2}$, que, segundo RAMALHO JÚNIOR et al. (1996), é chamada Lei de Boyle, sendo representada graficamente por uma curva (hipérbole eqüilátera). 
Conforme SISSOM \& PITTS (1988), todos os gases tendem a se comportar como um gás perfeito, quando submetidos a baixas pressões (até 1 atmosfera). A equação de gás perfeito conduz a bons resultados quando a densidade do gás for da ordem de um milésimo da densidade do líquido correspondente. Desse modo, em se tratando de um sistema envolvendo água e ar ou vapor d' água, conforme ocorre em instalações de bombeamento de água, sendo as massas específicas aproximadamente de $998 ; 1,22$ e de $0,8 \mathrm{~kg} \mathrm{~m}^{-3}$, respectivamente, presume-se que se pode aplicar a Lei de Boyle para a situação de escorvamento de bombas.

Segundo KARASSIK et al. (1986), o volume do tanque de escorva deve ser aproximadamente três vezes o volume da tubulação de sucção. Essa indicação coincide com o realizado na prática, pois os tanques de escorva vêm sendo muito utilizados em sistemas de irrigação em geral e também em sistemas de bombeamento de vinhaça no cultivo de cana-de-açúcar, com duas a três vezes o volume do tubo de sucção. Porém, na prática, também têm-se observado falhas de funcionamento dos tanques, não se conseguindo escorvar a instalação e ocorrendo até o esmagamento do tanque por falta de resistência ao vácuo.

Por essas razões, este estudo teve por objetivo realizar testes hidráulicos em laboratório, visando a estudar a aplicação da Lei de Boyle para o dimensionamento de tanques de escorva aplicáveis em instalações de bombeamento, analisando dois modelos de tanques.

\section{MATERIAL E MÉTODOS}

Na Figura 1, ilustra-se o esquema de instalação de bombeamento utilizando tanque de escorva.

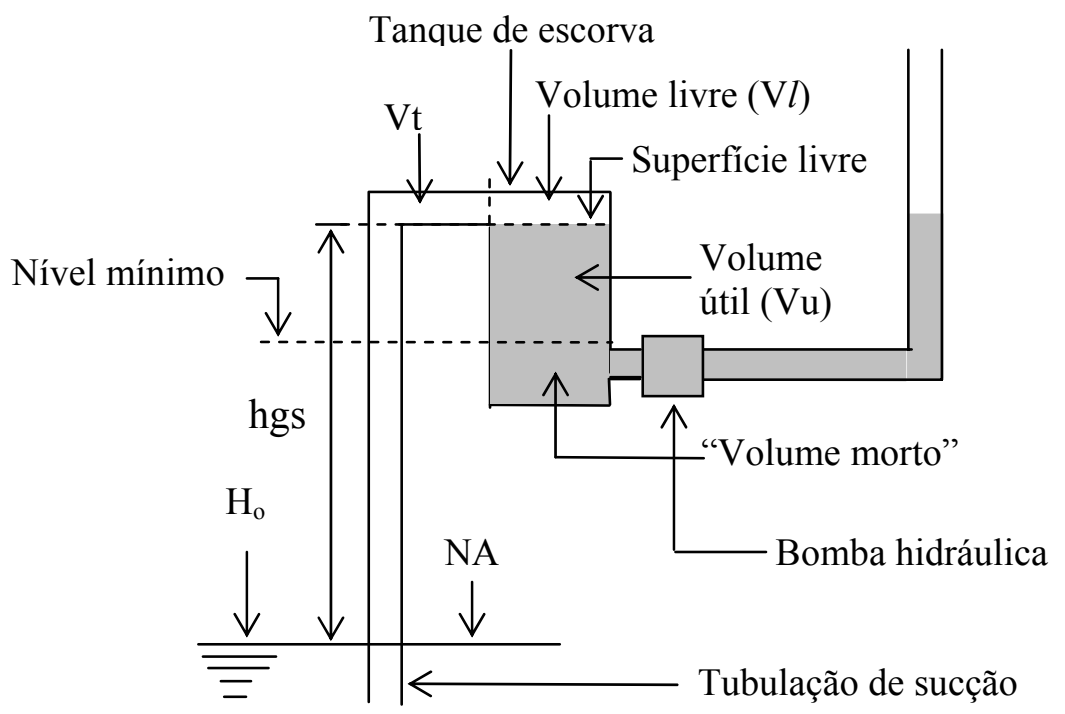

FIGURA 1. Esquema de uma instalação de bombeamento com tanque de escorva. Vt - volume da tubulação de sucção; hgs - altura geométrica de sucção; $\mathrm{H}_{0}$ - altura de coluna de água equivalente à pressão atmosférica local, e NA - nível da água na captação. Scheme of a pump with priming tank installation. $\mathrm{Vt}$ - suction pipe volume; hgs - geometric suction height; $\mathrm{H}_{0}$ - height of the water column equivalent to atmospheric pressure, and NA - level of water in the suction reservoir.

Para o funcionamento do sistema de bombeamento, primeiro deve-se fazer a escorva do conjunto, preenchendo-se com água a bomba e o tanque de escorva. A máxima altura de água que se consegue colocar no tanque coincide com o plano horizontal inferior do tubo de sucção, a partir do qual a água escoa para o reservatório de captação. Assim, pode-se definir como "superfície livre" o nível superior máximo da água no tanque e como "volume livre" o volume do tanque acima da superfície livre, que não fica preenchido com água.

Fazendo-se a partida do conjunto motobomba, inicia-se o esgotamento da água do tanque de escorva, e o limite de seu rebaixamento coincide com a posição do tubo de entrada da bomba, o 
qual pode ser definido como "nível mínimo". Quando a água chega ao nível mínimo, para que tenha ocorrido o escorvamento da tubulação de sucção, é necessário que a água que sobe pela mesma, tenha atingido a superfície livre. $\mathrm{O}$ volume vazio ( $\mathrm{ar}+$ vapor d'água) estabelecido no tanque será o volume livre mais o volume até o nível de rebaixamento da água no tanque. Assim, o volume do tanque deve ser calculado, levando-se em consideração que o enchimento do tubo de sucção, que incicialmente não estava escorvado, ocorre pelo deslocamento da água do tanque de escorva.

Para a determinação do volume útil do tanque $(\mathrm{Vu})$, aplicando-se a Lei de Boyle, considerando-se o local com altitude de $614 \mathrm{~m}$ (Jaboticabal - SP), com pressão atmosférica média de 9,65 metros de coluna d'água, tem-se: $\mathrm{P}_{1}=9,65$ m c.a.; $\mathrm{V}_{1}=\mathrm{Vt}+\mathrm{V} l ; \mathrm{e}, \mathrm{V}_{2}=\mathrm{V} l+\mathrm{Vu}$.

$\mathrm{O}$ valor de $\mathrm{P}_{2}$ dependerá da altura manométrica de sucção $(\mathrm{Hs})$, que é composta pela altura geométrica de sucção (hgs) somada à perda de carga na tubulação de sucção (hfs). Assim, a pressão absoluta $\left(\mathrm{P}_{2}\right)=$ pressão atmosférica - Hs. Portanto:

$$
\begin{aligned}
& 9,65(\mathrm{Vt}+\mathrm{V} l)=(9,65-\mathrm{Hs})(\mathrm{V} l+\mathrm{Vu}), \text { ou, } \\
& (\mathrm{V} l+\mathrm{Vu}) /(\mathrm{Vt}+\mathrm{V} l)=9,65 /(9,65-\mathrm{Hs})
\end{aligned}
$$

No presente estudo, foram realizadas avaliações de dois tanques de escorva, no Laboratório de Hidraúlica e Irrigação do Departamento de Engenharia Rural, da UNESP - Jaboticabal:

O tanque modelo 1 (Figura 2) apresenta formato cilíndrico, com a parte superior recurvada, com diâmetro de $0,46 \mathrm{~m}$ e altura de $0,49 \mathrm{~m}$. A entrada para a ligação da tubulação de sucção e a saída para o acoplamento da bomba são de tubos flangeados de $75 \mathrm{~mm}$ (3") de diâmetro. Na porção superior do tanque, encontram-se três aberturas de $25 \mathrm{~mm}$ (1") de diâmetro para instalação de vacuômetros e de válvula de abastecimento; na parte lateral do tanque, existem duas aberturas também de $25 \mathrm{~mm}$ (1”): uma para instalação de sensor de nível mínimo e outra para a drenagem e a limpeza do tanque. No interior do tanque, os tubos de entrada e saída do mesmo possuem curvas de $90^{\circ}$, de modo a reduzir o "volume livre" e o "volume morto" do tanque.

O tanque modelo 2 (Figura 3) apresenta formato cilíndrico, com as partes superior e inferior planas, diâmetro de $0,29 \mathrm{~m}$ e altura de $0,80 \mathrm{~m}$. A entrada para a ligação da tubulação de sucção e a saída para o acoplamento da bomba são com tubos de $63 \mathrm{~mm}(2,5$ ”) de diâmetro, com curvas de roscas internas. Em sua parte superior, o tanque possui uma abertura de $25 \mathrm{~mm}$ (1") de diâmetro para a instalação da válvula de abastecimento; acoplado à curva para ligação da tubulação de sucção, existe um tubo inserido verticalmente no interior do tanque, que permite manter o nível máximo da água próximo à superfície superior do tanque.
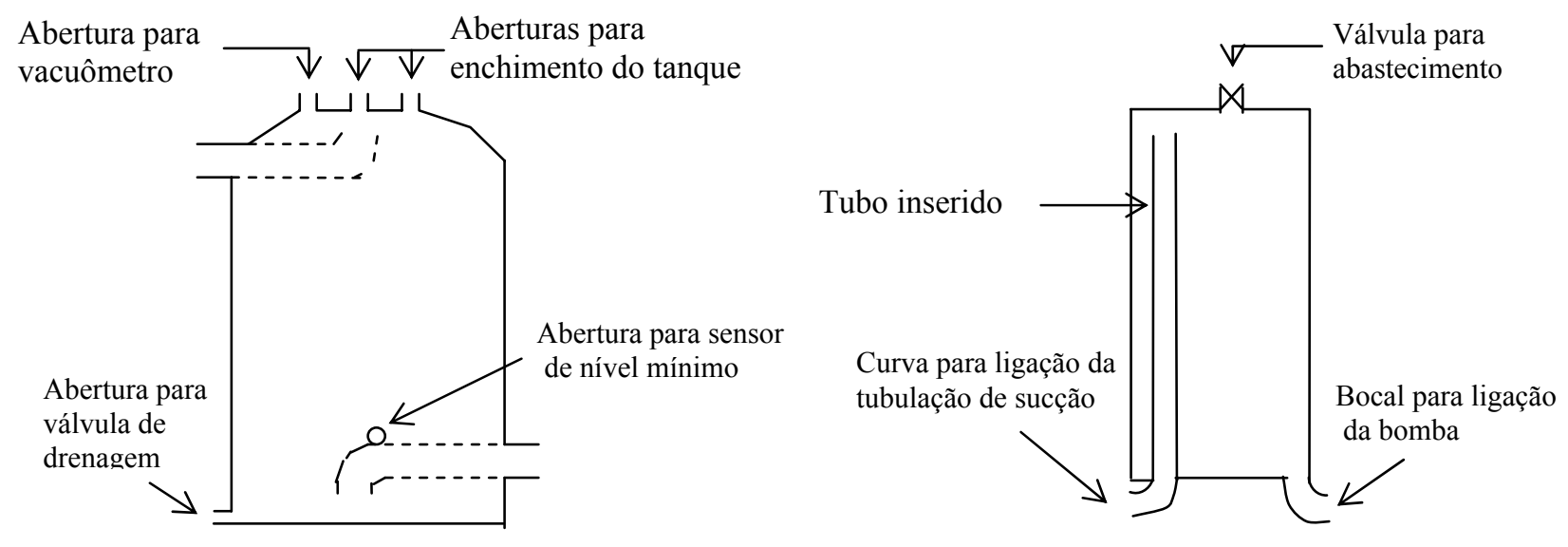

FIGURA 2. Esquema do tanque de escorva modelo 1. FIGURA 3. Esquema do tanque de escorva modelo 2. Scheme of the priming tank model 1. Scheme of the priming tank model 2.

As avaliações hidráulicas foram realizadas montando-se uma bancada de testes, com instalação dos reservatórios em um conjunto de bombeamento, conforme Figuras 4 e 5. 


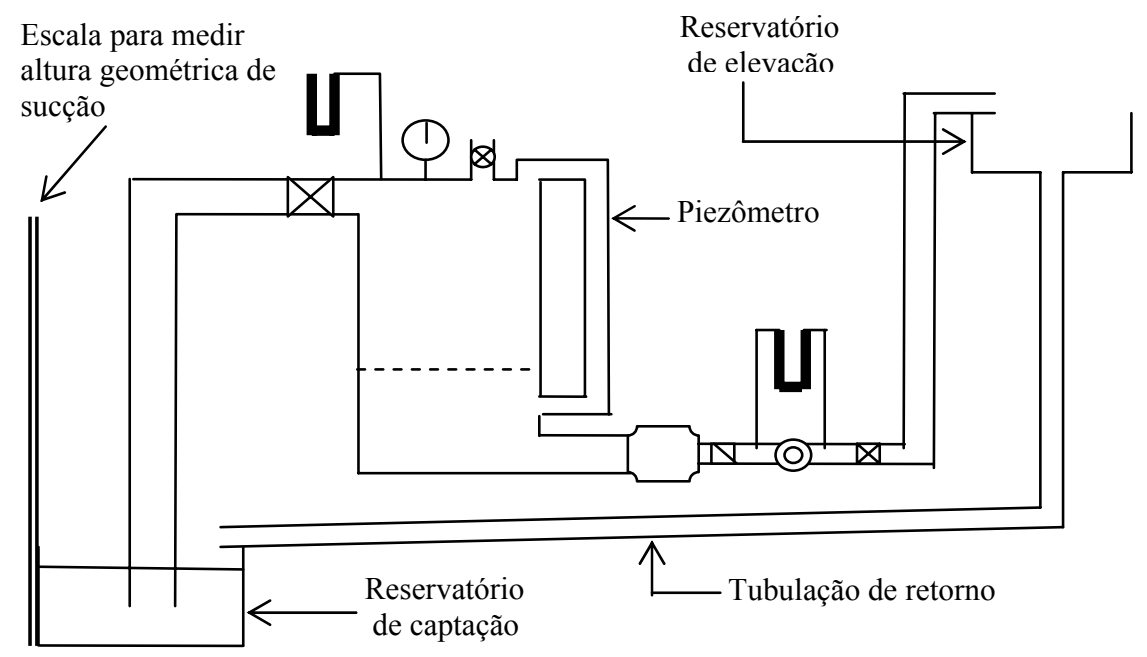

Legenda:

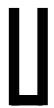

Manômetro (vacuômetro) de coluna de mercúrio

\ Válvula de gaveta

(1) Vacuômetro de Bourdon

(O) Placa de orifício para medição de vazão

Q Válvula para enchimento do reservatório

Bomba hidráulica

$\triangle$ Válvula de retenção

FIGURA 4. Esquema da montagem experimental para a avaliação do escorvamento utilizando tanques de escorva. Experimental scheme to evaluate the priming using tanks.

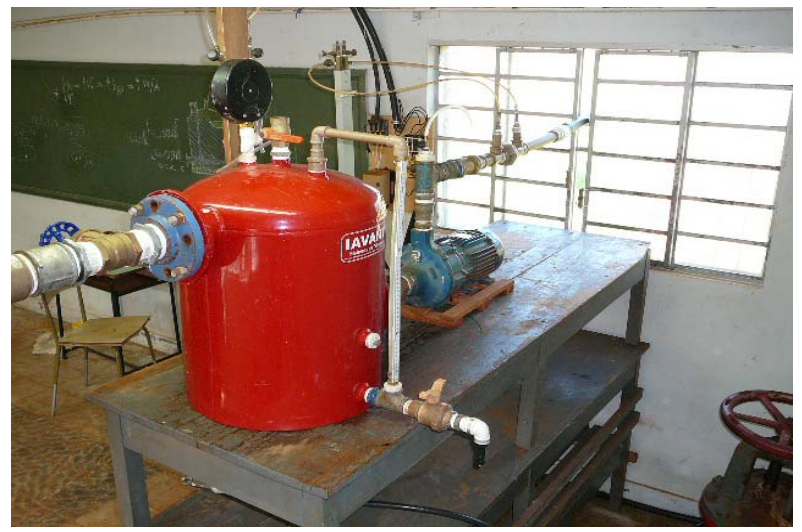

FIGURA 5. Montagem do tanque modelo 1 e posicionamento do piezômetro. Experimental installation of the tank model 1 and positioning of the gauge.

Foram utilizados os seguintes componentes na bancada de testes:

a) Escala para medir altura de sucção: constituída de trena com graduação em milímetros, com o valor zero coincidindo com o nível da superfície livre da água do tanque de escorva; com essa trena, mediu-se a altura geométrica de sucção (hgs), que se refere a distância vertical entre o zero da trena e o nível da água no reservatório de sucção.

b) Tubulação de sucção: para a avaliação do tanque 1, foi utilizado tubo de PVC, com diâmetro nomimal de $75 \mathrm{~mm}$ (3") e pressão nominal classificada como média, de 75 m.c.a. $(735 \mathrm{kPa})$; para o tanque 2, foi utilizado tubo de PVC, com diâmetro nominal de $50 \mathrm{~mm}$ (2") e pressão nominal de $75 \mathrm{~m}$ c.a. $(735 \mathrm{kPa})$. O comprimento total da tubulação de sucção foi variável, em função da altura geométrica de sucção. Devido ao espaço disponível para a instalação do sistema de bombeamento, para o tanque 1, os testes foram realizados com 2,16 m e 4,85 $\mathrm{m}$ de 
distância horizontais do centro do reservatório de sucção até a entrada do tanque; para o tanque 2, as distâncias horizontais foram de 3,18 m e 6,00 m.

c) Válvula de gaveta: com diâmetro de $50 \mathrm{~mm}$ (2"), permitiu aumentar a perda de carga na tubulação de sucção, para o estabelecimento dos valores de altura manométrica de sucção (Hs); a segunda válvula, colocada após a placa de orifício, foi utilizada para controle de vazão.

d) Vacuômetro de Bourdon: utilizado para a leitura direta da altura manométrica de sucção, facilitando o fechamento da válvula de gaveta até o valor de altura manométrica desejado em cada teste.

e) Vacuômetro de mercúrio: foi utilizado para calcular com extidão a altura manométrica de sucção, após a leitura do vacuômetro de Bourdon.

f) Piezômetro: é constituído de um duto transparente de plástico, colocando-se ao lado uma escala graduada em milímetros (Figura 5).

g) Conjunto motobomba: foi utilizada a bomba modelo KSB BLOC 32-125, rotor de $139 \mathrm{~mm}$ de diâmetro, com motor de $3 \mathrm{~kW}(4 \mathrm{cv})$, operando a $3.500 \mathrm{rpm}$.

h) Tubulação de recalque: é constituída de tubo de PVC de $40 \mathrm{~mm}$ (1,5”) de diâmetro, contendo uma válvula de retenção, um medidor de vazão constituído de placa de orifício e manômetro de mercúrio, e uma válvula de gaveta para controle de vazão. Após essa válvula, o tubo de recalque descarregava a vazão em uma caixa d'água a aproximadamente $2 \mathrm{~m}$ de altura em relação ao eixo da bomba.

i) Tubulação de retorno: é constituída de um duto flexível de polietileno de $50 \mathrm{~mm}$ (2”) de diâmetro, permitia o reabastecimento do reservatório de captação com a recirculação da água.

j) Reservatório de captação: é constituído de um tambor com 1,2 m de diâmetro e altura de $1,5 \mathrm{~m}$. Um piezômetro instalado na parede do tambor permitia verificar a altura da água no mesmo e a medição da altura geométrica de sucção, e

k) Placa de orifício: é construída utilizando-se de uma placa com perfuração de $16 \mathrm{~mm}$ de diâmetro (d), inserida em um tubo de PVC com $32 \mathrm{~mm}$ de diâmetro interno (D), definindo uma relação $\mathrm{d} / \mathrm{D}=0,50$. A placa foi inserida em uma luva de união de $\mathrm{PVC}$, de diâmetro nominal de $40 \mathrm{~mm}(1,5$ "), e as tomadas de pressão a montante e à jusante foram instaladas a $2 \mathrm{D}$ e a $8 \mathrm{D}$, respectivamente, conforme DELMÉE (1983). Anteriormente a sua utilização neste estudo, foi realizado um teste para a calibração da placa de orifício.

Para a avaliação dos tanques, utilizando-se da montagem esquematizada na Figura 4 e deslocando-se verticalmente o reservatório de captação, foram testadas quatro alturas geométricas de sucção: 1; 2; 3 e $4 \mathrm{~m}$. Para cada uma dessas alturas, a partida no conjunto motobomba foi realizada com a válvula de gaveta na saída da bomba totalmente fechada. Em seguida, abrindo-se gradualmente a válvula e observando-se o manômetro acoplado à placa de orifício, a vazão era regulada para $8 \mathrm{~m}^{3} \mathrm{~h}^{-1}$, de acordo com equação de regressão obtida com a curva de calibração do medidor. Esse valor de vazão foi adotado em função dos seguintes aspectos: compatibilidade com a curva vazão $x$ pressão da bomba, faixa de valores de velocidade normalmente recomendada em tubos de recalque e não provocar excessiva turbulência, desestabilizando o nível de água no reservatório de captação. Tendo-se estabelecido a abertura da válvula de gaveta, o conjunto era desligado, e nova partida era realizada com a válvula aberta. As avaliações foram realizadas com três repetições, com a seguinte rotina de procedimento:

a) Abrir a válvula de enchimento do tanque e completar o nível do mesmo até a superfície livre;

b) Ajustar o nível da água no reservatório de captação para a altura geométrica de sucção em estudo $(1 ; 2 ; 3$ ou $4 \mathrm{~m})$; 
c) Fechar a válvula de enchimento do tanque, ligar o conjunto motobomba e purgar o manômetro da placa de orifício;

d) Ajustar a vazão para $8 \mathrm{~m}^{3} \mathrm{~h}^{-1}$, atuando na válvula de gaveta no recalque e observando a coluna de mercúrio do manômetro da placa de orifício;

e) Reajustar o nível do reservatório de captação;

f) Aguardar aproximadamente 2 minutos para equilibrar o sistema, fazendo novo ajuste da vazão, se necessário;

g) Estabelecer a altura manométrica de sucção, atuando na válvula de gaveta da sucção e com leituras do vacuômetro de Bourdon e de coluna de mercúrio. Uma vez que a altura manométrica afeta a vazão, realizar novo ajuste da vazão;

h) Ler a escala do piezômetro do tanque, permitindo obter o volume de rebaixamento no mesmo;

i) Observar se a instalação mantém o escorvamento sem esgotar o tanque além do nível mínimo, ou se entra em cavitação, e

j) Desligar o conjunto e realizar as etapas de a a j, fazendo-se três repetições.

Baseando-se na eq.(1), para cada uma das quatro alturas geométricas, foram avaliadas as possíveis relações $(\mathrm{Vu}+\mathrm{V} l) /(\mathrm{Vt}+\mathrm{V} l)$, para as diversas alturas manométricas de sucção, obtidas com as leituras dos vacuômetros. Assim, a primeira altura manométrica avaliada foi a existente com a válvula de gaveta totalmente aberta na tubulação de sucção. Em seguida, fechando-se essa válvula, foram avaliadas as demais alturas manométricas, com valores crescentes de $1 \mathrm{~m}$, até queda do escorvamento ou cavitação da bomba. Uma vez que a altura manométrica da tubulação de sucção afeta a vazão, para cada valor de altura manométrica testado, foram manobradas conjuntamente as válvulas de gaveta na sucção e no recalque, para manter a vazão em $8 \mathrm{~m}^{3} \mathrm{~h}^{-1}$, observando-se a coluna de mercúrio do manômetro da placa de orifício.

De acordo com a eq.(1), para a obtenção da relação $(\mathrm{Vu}+\mathrm{V} l) /(\mathrm{Vt}+\mathrm{V} l)$, além da altura manométrica, foi determinado o volume da tubulação de sucção, para os quatro valores de altura geométrica estudados. Para isso, antes de iniciar os testes, a tubulação foi preenchida e, em seguida, esvaziada, coletando-se e pesando-se o volume de água do interior do tubo.

\section{RESULTADOS E DISCUSSÃO}

\section{Valores obtidos para o cálculo da relação $(\mathrm{Vu}+\mathrm{V} l) /(\mathrm{Vt}+\mathrm{V} l)$}

Com os dados coletados durante os testes hidráulicos, foram calculadas as médias das relações $(\mathrm{Vu}+\mathrm{V} l) /(\mathrm{Vt}+\mathrm{V} l)$ para as diferentes alturas manométricas $(\mathrm{Hs})$ (Tabelas 1 e 2). Com essas médias, foram obtidas as curvas e equações de regressões para os dois tanques (Figuras 6 e 7). Nessas figuras, verifica-se que os valores das relações $(\mathrm{Vu}+\mathrm{V} l) /(\mathrm{Vt}+\mathrm{V} l)$ calculados com os dados obtidos nos testes hidráulicos apresentaram a mesma tendência das relações obtidas pela Lei de Boyle, ou seja, aumentaram em função da altura manométrica Hs. Desse modo, conhecendo-se Vl, Vt e Hs, pode-se calcular o volume do tanque $(\mathrm{Vu})$, que depende fundamentalmente de $\mathrm{Vt}$ e Hs. Entretanto, pode-se verificar, também, que as relações $(\mathrm{Vu}+\mathrm{V} l) /(\mathrm{Vt}+\mathrm{V} l)$ obtidas com os valores experimentais foram sempre superiores às relações obtidas pela Lei de Boyle.

Para comparar os valores obtidos experimentalmente com os valores obtidos pela Lei de Boyle, foi realizada a análise estatística (Tabelas 3 e 4) segundo MASSEY \& DIXON (1969), empregando-se o programa ESTAT, desenvolvido pelo Departamento de Ciências Exatas, da UNESP - Jaboticabal. 
TABELA 1. Médias das relações $(\mathrm{Vu}+\mathrm{V} l) /(\mathrm{Vt}+\mathrm{V} l)$ obtidas para o tanque modelo 1 avaliado com diferentes valores de hgs, Hs e de LH. Medium of the relations $(\mathrm{Vu}+\mathrm{VI}) /(\mathrm{Vt}+\mathrm{VI})$ obtained for the tank model 1 evaluated with different values of hgs, $\mathrm{Hs}$ and $\mathrm{LH}$.

\begin{tabular}{|c|c|c|c|c|c|c|c|c|c|c|c|c|c|c|c|c|}
\hline \multicolumn{6}{|c|}{$\mathrm{LH}=4,85 \mathrm{~m}$} & \multicolumn{8}{|c|}{$\mathrm{LH}=2,16 \mathrm{~m}$} & \multirow{2}{*}{\multicolumn{3}{|c|}{ Médias }} \\
\hline \multicolumn{2}{|c|}{ hgs $=1 \mathrm{~m}$} & \multicolumn{2}{|c|}{ hgs $=2 \mathrm{~m}$} & \multicolumn{2}{|c|}{ hgs $=3 \mathrm{~m}$} & \multicolumn{2}{|c|}{ hgs $=1 \mathrm{~m}$} & \multicolumn{2}{|c|}{ hgs $=2 \mathrm{~m}$} & \multicolumn{2}{|c|}{ hgs $=3 \mathrm{~m}$} & \multicolumn{2}{|c|}{$\mathrm{hgs}=4 \mathrm{~m}$} & & & \\
\hline $\begin{array}{c}\text { Hs } \\
\text { (m c.a.) }\end{array}$ & $\frac{\mathrm{Vu}+\mathrm{Vl}}{\mathrm{Vt}+\mathrm{V} l}$ & $\begin{array}{c}\text { Hs } \\
\text { (m c.a.) }\end{array}$ & $\frac{\mathrm{Vu}+\mathrm{Vl}}{\mathrm{Vt}+\mathrm{Vl}}$ & $\begin{array}{c}\text { Hs } \\
\text { (m c.a.) }\end{array}$ & $\frac{\mathrm{Vu}+\mathrm{Vl}}{\mathrm{Vt}+\mathrm{Vl}}$ & $\begin{array}{c}\text { Hs } \\
\text { (m c.a.) }\end{array}$ & $\frac{\mathrm{Vu}+\mathrm{Vl}}{\mathrm{Vt}+\mathrm{V} l}$ & $\begin{array}{c}\text { Hs } \\
\text { (m c.a.) }\end{array}$ & $\frac{\mathrm{Vu}+\mathrm{Vl}}{\mathrm{Vt}+\mathrm{V} l}$ & $\begin{array}{c}\text { Hs } \\
\text { (m c.a.) }\end{array}$ & $\frac{\mathrm{Vu}+\mathrm{Vl}}{\mathrm{Vt}+\mathrm{Vl}}$ & $\begin{array}{c}\text { Hs } \\
\text { (m c.a.) }\end{array}$ & $\frac{\mathrm{Vu}+\mathrm{Vl}}{\mathrm{Vt}+\mathrm{Vl}}$ & $\begin{array}{c}\text { Hs } \\
\text { (m c.a.) }\end{array}$ & $\frac{\mathrm{Vu}+\mathrm{V} l}{\mathrm{Vt}+\mathrm{V} l}$ & $\frac{9,65}{(9,65-\mathrm{Hs})}$ \\
\hline 1,169 & 1,262 & & & & & 1,146 & 1,203 & & & & & & & 1,157 & 1,232 & 1,136 \\
\hline 2,165 & 1,472 & 2,197 & 1,447 & & & 2,147 & 1,384 & 2,170 & 1,383 & & & & & 2,170 & 1,422 & 1,290 \\
\hline 3,076 & 1,714 & 3,035 & 1,700 & 3,130 & 1,591 & 3,176 & 1,630 & 3,180 & 1,646 & 3,176 & 1,632 & & & 3,129 & 1,652 & 1,480 \\
\hline 4,027 & 2,010 & 4,009 & 1,879 & 4,023 & 1,695 & 4,036 & 1,960 & 4,041 & 1,961 & 4,018 & 1,930 & 4,086 & 1,920 & 4,034 & 1,908 & 1,718 \\
\hline 4,978 & 2,174 & 4,992 & 1,926 & 5,019 & 1,695 & 5,010 & 2,505 & 5,006 & 2,390 & 4,992 & 2,332 & 5,001 & 2,053 & 5,000 & 2,153 & 2,075 \\
\hline
\end{tabular}

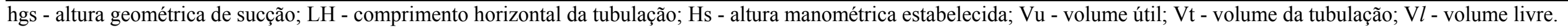

TABELA 2. Médias das relações $(\mathrm{Vu}+\mathrm{V} l) /(\mathrm{Vt}+\mathrm{V} l)$ obtidas para o tanque modelo 2 avaliado com diferentes valores de hgs, Hs e de LH. Medium of the relations $(\mathrm{Vu}+\mathrm{Vl}) /(\mathrm{Vt}+\mathrm{Vl})$ obtained for the tank model 2 evaluated with different values of hgs, Hs and LH.

\begin{tabular}{|c|c|c|c|c|c|c|c|c|c|c|c|c|c|c|c|c|}
\hline \multicolumn{6}{|c|}{$\mathrm{LH}=6,00 \mathrm{~m}$} & \multicolumn{8}{|c|}{$\mathrm{LH}=3,18 \mathrm{~m}$} & \multirow{2}{*}{\multicolumn{3}{|c|}{ Médias }} \\
\hline \multicolumn{2}{|c|}{$\mathrm{hgs}=1 \mathrm{~m}$} & \multicolumn{2}{|c|}{$\mathrm{hgs}=2 \mathrm{~m}$} & \multicolumn{2}{|c|}{ hgs $=3 \mathrm{~m}$} & \multicolumn{2}{|c|}{$\mathrm{hgs}=1 \mathrm{~m}$} & \multicolumn{2}{|c|}{$\mathrm{hgs}=2 \mathrm{~m}$} & \multicolumn{2}{|c|}{$h g s=3 \mathrm{~m}$} & \multicolumn{2}{|c|}{$\mathrm{hgs}=4 \mathrm{~m}$} & & & \\
\hline $\begin{array}{c}\text { Hs } \\
\text { (m c.a ) }\end{array}$ & $\frac{\mathrm{Vu}+\mathrm{Vl}}{\mathrm{Vt}+\mathrm{Vl}}$ & $\begin{array}{c}\text { Hs } \\
\text { (m c.a.) }\end{array}$ & $\frac{\mathrm{Vu}+\mathrm{Vl}}{\mathrm{Vt}+\mathrm{Vl}}$ & $\begin{array}{c}\text { Hs } \\
\text { (m c.a.) }\end{array}$ & $\frac{\mathrm{Vu}+\mathrm{V} l}{\mathrm{Vt}+\mathrm{V} l}$ & $\begin{array}{c}\text { Hs } \\
\text { (m c.a.) }\end{array}$ & $\frac{\mathrm{Vu}+\mathrm{Vl}}{\mathrm{Vt}+\mathrm{Vl}}$ & $\begin{array}{c}\text { Hs } \\
\text { (m c.a.) }\end{array}$ & $\frac{\mathrm{Vu}+\mathrm{V} l}{\mathrm{Vt}+\mathrm{V} l}$ & $\begin{array}{c}\text { Hs } \\
\text { (m c.a.) }\end{array}$ & $\frac{\mathrm{Vu}+\mathrm{V} l}{\mathrm{Vt}+\mathrm{V} l}$ & $\begin{array}{c}\text { Hs } \\
\text { (m c.a.) }\end{array}$ & $\frac{\mathrm{Vu}+\mathrm{Vl}}{\mathrm{Vt}+\mathrm{V} l}$ & $\begin{array}{c}\text { Hs } \\
\text { (m c.a.) }\end{array}$ & $\frac{\mathrm{Vu}+\mathrm{Vl}}{\mathrm{Vt}+\mathrm{V} l}$ & $\frac{9,65}{9,65-\mathrm{Hs}}$ \\
\hline 1,436 & 1,495 & & & & & 1,323 & 1,660 & & & & & & & 1,379 & 1,577 & 1,167 \\
\hline 2,487 & 1,754 & 2,487 & 1,722 & & & 2,129 & 1,981 & 2,442 & 1,746 & & & & & 2,386 & 1,801 & 1,329 \\
\hline 3,058 & 1,908 & 3,058 & 1,887 & 3,529 & 1,905 & 3,162 & 2,444 & 3,176 & 2,002 & 3,488 & 2,008 & & & 3,245 & 2,026 & 1,507 \\
\hline 4,063 & 2,169 & 4,041 & 2,152 & 4,068 & 1,964 & 4,036 & 2,952 & 4,068 & 2,363 & 4,050 & 2,260 & 4,544 & 2,258 & 4,124 & 2,303 & 1,746 \\
\hline 5,028 & 2,113 & 5,015 & 1,917 & 5,037 & 2,032 & 5,015 & 3,072 & 5,037 & 2,650 & 5,055 & 2,662 & 5,060 & 2,501 & 5,035 & 2,421 & 2,091 \\
\hline
\end{tabular}

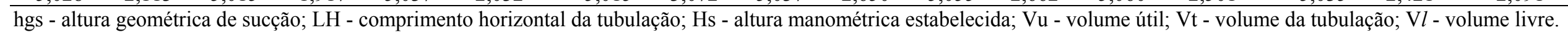




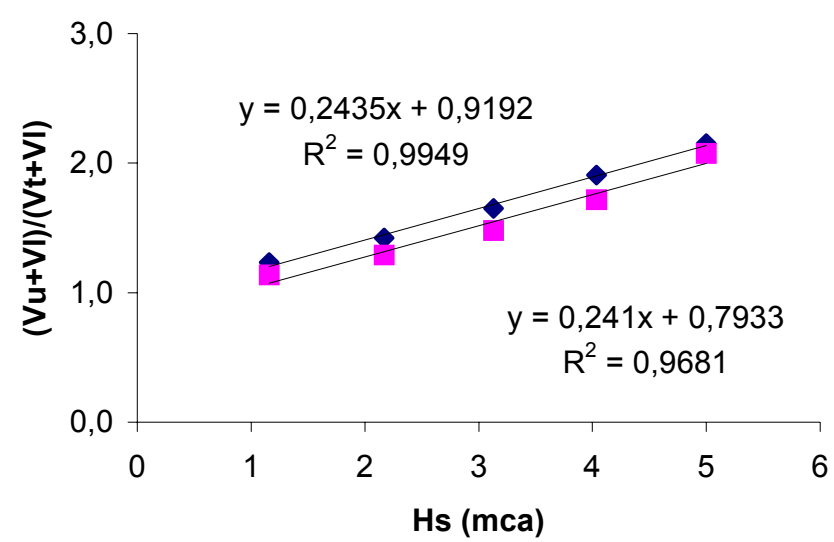

- Valores Obtidos

Lei de Boyle

FIGURA 6. Curvas e equações de regressão das relações $(\mathrm{Vu}+\mathrm{V} l) /(\mathrm{Vt}+\mathrm{V} l)$ em função da altura manométrica de sucção (Hs), obtidas pela equação de Boyle e nos testes hidráulicos, para o tanque modelo 1. Curves and regression equations of the relations $(\mathbf{V u}+\mathrm{Vl}) /(\mathrm{Vt}+\mathrm{Vl})$ according to the suction head (Hs), obtained by Boyle's equation and by hydraulic tests for the tank model 1.

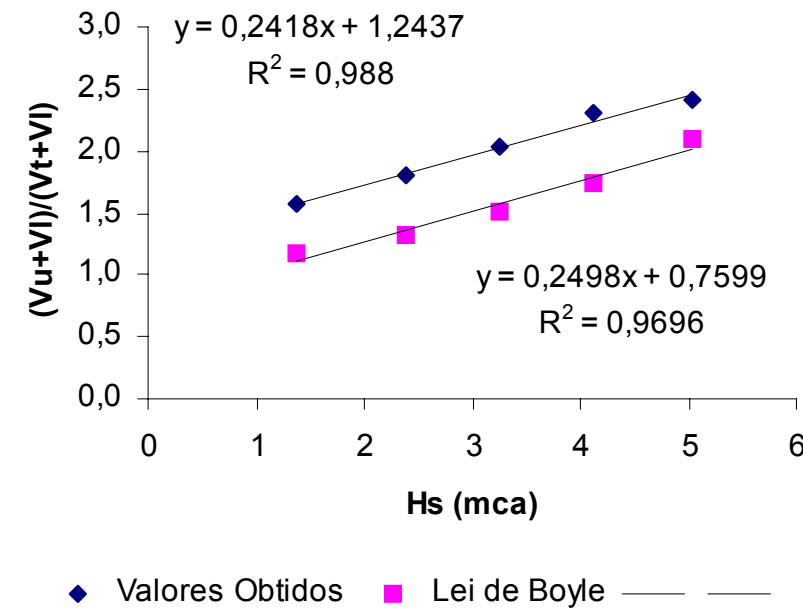

FIGURA 7. Curvas e equações de regressão das relações $(\mathrm{Vu}+\mathrm{V} l) / \mathrm{Vt}+\mathrm{V} l)$ em função da altura manométrica de sucção (Hs), obtidas pela equação de Boyle e nos testes hidráulicos, para o tanque modelo 2. Curves and regression equations of the relations $(\mathrm{Vu}+\mathrm{Vl}) /(\mathrm{Vt}+\mathrm{Vl})$ according to the suction head (Hs), obtained by Boyle's equation and by hydraulic tests for the tank model 2.

A comparação estatística revelou que as regressões obtidas para os dois modelos de tanques foram significativas $(\mathrm{p}<0,01)$ para o modelo linear, com coeficientes de correlação 0,9974 e 0,9939. Como o modelo linear foi significativo, foram comparados os coeficientes lineares e angulares das equações das retas obtidas com valores experimentais e com a Lei de Boyle, estabelecendo a hipótese da nulidade $\mathrm{H}_{0}$, de que as retas são não-coincidentes e não-paralelas.

Para o tanque 1 , o teste para coincidência foi significativo $(\mathrm{p}<0,05)$, e, portanto, as duas retas não podem ser consideradas coincidentes. Porém, o teste de paralelismo apresentou resultado nãosignificativo, e, portanto, as duas retas podem ser consideradas paralelas. Para o tanque 2, semelhantemente ao tanque 1 , o teste de coincidência foi significativo $(p<0,01)$ e não-significativo para o paralelismo. Assim, as retas são paralelas, porém, não-coincidentes.

TABELA 3. Análise estatística referente aos dados experimentais e aos calculados pela equação de Boyle para o tanque modelo 1 . Statistical analysis of the experimental data and calculated by Boyle's equation for the tank model 1.

\begin{tabular}{|c|c|c|c|c|c|c|c|c|c|c|c|c|}
\hline \multirow{2}{*}{$\begin{array}{c}\text { Tanque } 1 \\
\text { Causas de } \\
\text { variação }\end{array}$} & \multicolumn{4}{|c|}{ Reta 1} & \multicolumn{4}{|c|}{ Reta 2} & \multicolumn{4}{|c|}{ Reta $1 \times$ Reta 2} \\
\hline & GL & SQ & QM & $\mathrm{F}$ & GL & SQ & QM & $\mathrm{F}$ & GL & SQ & QM & $\mathrm{F}$ \\
\hline Regressão Linear & 1 & 0,5408 & 0,5408 & $584,40^{* *}$ & 1 & 0,5298 & 0,5298 & $91,09^{* *}$ & 1 & 1,0705 & 1,0705 & $132,01 * *$ \\
\hline $\begin{array}{c}\text { Desvios de } \\
\text { regressão }\end{array}$ & 3 & 0,0028 & 0,0009 & & 3 & 0,0174 & 0,0058 & & 8 & 0,6459 & 0,0081 & \\
\hline Total & 4 & 0,5435 & & & 4 & 0,5472 & & & 9 & 1,1354 & & \\
\hline \multicolumn{13}{|c|}{ Estimativa de parâmetros da Equação de Regressão $(\mathrm{Y}=\mathrm{AX}+\mathrm{B})$} \\
\hline & A & 0,2435 & EPA & 0,0101 & A & 0,2410 & EPA & 0,0252 & A & 0,2422 & EPA & 0,0211 \\
\hline & $\mathrm{B}$ & 0,9192 & EPB & 0,0340 & $\mathrm{~B}$ & 0,7933 & EPB & 0,0853 & B & 0,8562 & EPB & 0,0712 \\
\hline \multicolumn{13}{|c|}{ Coeficiente de correlação e teste } \\
\hline & $\mathrm{R}$ & 0,9974 & $\mathrm{~T}$ & $24,174 * *$ & $\mathrm{R}$ & 0,9839 & $\mathrm{~T}$ & $9,544^{* *}$ & $\mathrm{R}$ & 0,9710 & $\mathrm{~T}$ & $11,490 * *$ \\
\hline
\end{tabular}

Teste $\mathrm{F}$ para coincidência: $\mathrm{F}=6,62^{*}$

Teste $\mathrm{T}$ para paralelismo: $\mathrm{T}=0,09 \mathrm{NS}$ 
TABELA 4. Análise estatística referente aos dados experimentais e aos calculados pela equação de Boyle para o tanque modelo 2. Statistical analysis of the experimental data and calculated by Boyle's equation for the tank model 2.

\begin{tabular}{lcccccccccccc}
\hline \multicolumn{1}{c}{ Tanque 2 } & \multicolumn{3}{c}{ Reta 1 } & \multicolumn{4}{c}{ Reta 2 } & \multicolumn{4}{c}{ Reta 1 x Reta 2 } \\
\hline Causas de variação & GL & SQ & QM & F & GL & SQ & QM & F & GL & SQ & QM & F \\
\hline $\begin{array}{l}\text { Regressão Linear } \\
\begin{array}{c}\text { Desvios de } \\
\text { regressão }\end{array}\end{array}$ & 1 & 0,4791 & 0,4791 & $245,17^{* *}$ & 1 & 0,5109 & 0,5109 & $95,77^{* *}$ & 1 & 0,9897 & 0,9897 & $14,51^{* *}$ \\
\hline Total & 4 & 0,0059 & 0,0020 & & 3 & 0,0160 & 0,0053 & & 8 & 0,5456 & 0,0682 & \\
\hline
\end{tabular}

Estimativa de parâmetros da Equação de Regressão $(\mathrm{Y}=\mathrm{AX}+\mathrm{B})$

\begin{tabular}{cccccccccccc} 
A & 0,2418 & EPA & 0,0154 & A & 0,2497 & EPA & 0,0255 & A & 0,2457 & EPA & 0,0682 \\
B & 1,2437 & EPB & 0,0537 & B & 0,7607 & EPB & 0,0887 & B & 1,0022 & EPB & 0,2243 \\
\hline \multicolumn{10}{c}{ Coeficiente de correlação e teste } \\
\hline R & 0,9939 & T & $15,658^{* *}$ & R & 0,9847 & T & $9,786^{* *}$ & R & 0,8029 & T & $3,809^{* *}$ \\
\hline
\end{tabular}

Teste $\mathrm{F}$ para coincidência: $\mathrm{F}=71,86^{* *}$

Teste $\mathrm{T}$ para paralelismo: $\mathrm{T}=-0,26 \mathrm{NS}$

Como apresentado nas Figuras 6 e 7 e pelas análises estatísticas realizadas, verifica-se que as relações $(\mathrm{Vu}+\mathrm{V} l) /(\mathrm{Vt}+\mathrm{V} l)$ obtidas experimentalmente são superiores às previstas, utilizando-se da Lei de Boyle, conforme percentagens apresentadas na Tabela 5.

TABELA 5. Diferenças percentuais entre os valores médios das relações $(\mathrm{Vu}+\mathrm{V} l) /(\mathrm{Vt}+\mathrm{V} l)$ obtidas experimentalmente e pela lei de Boyle. Differences between the average percentage of relations $(\mathrm{Vu}+\mathrm{Vl}) /(\mathrm{Vt}+\mathrm{Vl})$ obtained experimentally and by the Boyle's law.

\begin{tabular}{cccccccc}
\hline \multicolumn{3}{c}{ Tanque Modelo 1 } & \multicolumn{4}{c}{ Tanque Modelo 2 } \\
\hline Hs (mca) & $\begin{array}{c}\text { Valores } \\
\text { Experimentais }\end{array}$ & Lei de Boyle & $\begin{array}{c}\text { Diferença } \\
(\%)\end{array}$ & Hs (mca) & $\begin{array}{c}\text { Valores } \\
\text { Experimentais }\end{array}$ & $\begin{array}{c}\text { Lei de } \\
\text { Boyle }\end{array}$ & $\begin{array}{c}\text { Diferença } \\
(\%)\end{array}$ \\
\hline 1,157 & 1,232 & 1,136 & 8,5 & 1,379 & 1,577 & 1,167 & 35,2 \\
2,170 & 1,422 & 1,290 & 10,2 & 2,386 & 1,801 & 1,329 & 35,5 \\
3,129 & 1,652 & 1,480 & 11,6 & 3,245 & 2,026 & 1,507 & 34,5 \\
4,034 & 1,908 & 1,718 & 11,1 & 4,124 & 2,303 & 1,746 & 31,9 \\
5,000 & 2,153 & 2,075 & 3,8 & 5,035 & 2,421 & 2,091 & 15,8 \\
\hline Média & \multicolumn{7}{c}{ Média } \\
\hline
\end{tabular}

Pela Tabela 5, verifica-se que, em média, os valores de relação $(\mathrm{Vu}+\mathrm{V} l) /(\mathrm{Vt}+\mathrm{V} l)$ obtidos experimentalmente foram de $9,0 \%$ e $30,6 \%$ superiores aos previstos pela Lei de Boyle. Essa discrepância entre os resultados, provavelmente, ocorre porque nas condições de bombeamento estudadas existem ar e vapor d'água, diferindo da situação de gás ideal, preconizada pela Lei de Boyle.

As maiores diferenças das relações $(\mathrm{Vu}+\mathrm{V} l) /(\mathrm{Vt}+\mathrm{V} l)$ em relação à Lei de Boyle, possivelmente, também podem ser explicadas pelo procedimento experimental realizado. Como a calibração do volume de ar nos tanques foi realizada esvaziando-os de litro em litro, relacionandose com o nível da água no piezômetro, durante o bombeamento, em função do formato do tanque, o volume livre e o volume útil $(\mathrm{Vu})$ podem ter permanecido parcialmente ocupados, não sendo identificados no piezômetro. Assim, é possível que a soma $\mathrm{Vu}+\mathrm{V} l$ durante os testes seja menor do que os valores anotados. Também, o formato do tanque pode afetar na distribuição de água ao mesmo, pois o tubo inserido faz com que a água atinja sua parte superior e escorra pelas laterais até definir o nível minímo identificado no piezômetro. Dessa forma, o tanque modelo 2 apresentou maiores diferenças entre os valores experimentalmente obtidos e os previstos pela Lei de Boyle, com diferença média de aproximadamente $30 \%$. 
Tendo-se constatado que os valores experimentais são maiores que os previstos teoricamente, no dimensionamento de tanques de escorva, deve-se prever percentagem de acréscimo e procurar fabricá-los com formato que proporcione volume livre mínimo. Segundo VILLA NOVA et al. (1997), o dimensionamento do tanque de escorva pode ser baseado totalmente na Lei de Boyle, porém a indicação desses autores foi sustentada apenas na teoria da equação geral dos gases. $\mathrm{Na}$ prática, os resultados da presente pesquisa indicaram que se deve fazer acréscimo do volume previsto para o dimensionamento do tanque.

\section{CONCLUSÕES}

O volume do tanque de escorva é função do volume da tubulação de sucção da instalação de bombeamento e da altura manométrica de sucção;

No cálculo do volume do tanque de escorva pela Lei de Boyle, deve-se prever percentagem de acréscimo, que, neste estudo, foi de $9 \%$ para um modelo de tanque e de $30 \%$ para o outro modelo.

Os dados obtidos para os dois modelos de tanque, comparados com os dados estimados pela Lei de Boyle, resultaram diferenças, sugerindo que outros fatores podem influenciar no dimensionamento, tal como o formato e a posição do tanque, e precisam ser estudados.

\section{REFERÊNCIAS}

AZEVEDO NETTO, J.M.; FERNANDES, M.F.; ARAUJO, R.de; ITO, A.E. Manual de hidráulica. 8.ed. São Paulo: Edgard Blücker, 1998. 670 p.

CARVALHO, D.F. Instalações elevatórias - bombas. 5.ed. Belo Horizonte: FUMARC, 1992. 352 p.

DELMÉE, G.J. Manual de medição de vazão. São Paulo: Edgard Blücher, 1983. 476 p.

KARASSIK, I.J.; KRUTZSCH, W.C.; FRASER, W.H.; MESSINA, J.P. Pump handbook. $2^{\text {nd }}$ ed. New York: McGraw-Hill, 1986. 1.351 p.

MASSEY, F.J.; DIXON, W.J. Introduction to statistical analysis. New York: McGraw-Hill, 1969. $370 \mathrm{p}$.

MACINTYRE, A.J. Bombas e instalações de bombeamento.2.ed. Rio de Janeiro: LTC, 1997. 782 p.

RAMALHO JÚNIOR, F.; FERRARO, N.G.; SOARES, P.A.de T. Os fundamentos da física. 6.ed. São Paulo: Moderna, 1996. v.2, 463 p.

SISSOM, L.E.; PITTS, D.R. Fenômenos de transporte. Rio de Janeiro: Guanabara, 1988. 765 p.

VILLA NOVA, N.A.; TOMAZELA, C.; DOURADO NETO, D.; FANCELLI, A.L.

Dimensionamento de um balão de escorva. In: TECNOLOGIA DE PRODUÇÃO DO FEIJÃO

IRRIGADO, 1997, Piracicaba. Anais... Piracicaba: FEALQ - USP, 1997. p.159-62.

VILELA, L.A.A.; BOTREL, T.A.; SOCCOL, O.J.; GERVÁSIO, E.S.; NETO, O.R. Dispositivo para monitoramento da operação de conjuntos motobomba elétricos. Engenharia Agrícola, Jaboticabal, v.23, n.2, 2003, p. 250-6. 